\title{
Identification of a mitochondrial 12S rRNA A1555G mutation causing aminoglycoside-induced deafness in a large Thai family
}

Pongsathorn Chaiyasapa ${ }^{\mathrm{a}}$, Chalurmpon Srichomthong ${ }^{\mathrm{b}, \mathrm{c}}$, Siraprapa Tongkobpetch ${ }^{\mathrm{b}, \mathrm{c}}$, Kanya Suphapeetiporn ${ }^{\mathrm{b}, \mathrm{c}}$, Vorasuk Shotelersuk ${ }^{\mathrm{b}, \mathrm{c}}$

anterdepartment of Biomedical Sciences, Faculty of Graduate School, Chulalongkorn University, Bangkok 10330, Thailand

${ }^{b}$ Center of Excellence for Medical Genetics, Department of Pediatrics, Faculty of Medicine, Chulalongkorn University, Bangkok 10330, Thailand

'Excellence Center for Medical Genetics, King Chulalongkorn Memorial Hospital, the Thai Red Cross, Bangkok 10330, Thailand

\begin{abstract}
Background: Hearing loss is among the most frequent sensory disorders. Preventable causes include medications given to genetically susceptible individuals. Several families around the world with an A1555G mitochondrial mutation who became profoundly deaf after receiving aminoglycosides have been described. However, none has been reported in Thailand.

Objectives: To identify the cause of hearing loss of a large Thai family with 11 members who reportedly turned deaf after receiving antibiotics.

Methods: We obtained blood samples from 5 members; 4 of whom had hearing loss. Mutation analyses were performed using molecular techniques including polymerase chain reaction, Sanger sequencing, and restriction fragment length polymorphism.

Results: All 4 affected members were found to harbor the same A1555G mitochondrial mutation, while the unaffected had only the wild-type A.

Conclusions: We have identified the mitochondrial mutation leading to aminoglycoside-induced hearing loss in a Thai population. Raising awareness for medical practitioners of this genetic susceptibility in Thailand is warranted. Avoidance of certain medications in these individuals would prevent this acquired permanent hearing loss.
\end{abstract}

Keywords: Aminoglycoside, deafness, mitochondrial DNA, MT-RNR1, A1555G, mutation analysis, Thai.

Hearing loss is one of the most frequent sensory disorders. It affects approximately one in 700-1000 newborns $[1,2]$. It can be the result of genetic mutations or environmental factors, which include ototoxic drugs such as aminoglycoside antibiotics [2]. These types of antibiotics are widely used because of their effectiveness in the treatment of gramnegative infections. They are also easily accessible and inexpensive to produce [3]. Examples of aminoglycosides include streptomycin, neomycin,

Correspondence to: Kanya Suphapeetiporn, Division of Medical Genetics and Metabolism, Department of Pediatrics, Faculty of Medicine, Chulalongkorn University, Bangkok 10330, Thailand. E-mail: kanya.su@chula.ac.th kanamycin, paromomycin, gentamicin, tobramycin, amikacin, netilmicin, and spectinomycin [4]. They are usually safe to use within recommended limits. However, they are known causes of ototoxicity [4]. For certain genetically susceptible individuals, receiving these medications could result in permanent sensorineural hearing loss. There have been several reports of profound hearing loss in family members receiving aminoglycosides [5-8]. These patients were found to harbor a maternally inherited A1555G mutation located at a highly conserved region of the mitochondrial encoded 12S rRNA gene (MT-RNR1) in the mitochondrial genome. In these patients, the standard dose of aminoglycosides can cause irreversible deafness. 
Here, we identified a large Thai family with 11 members reported to have postlingual deafness after receiving aminoglycosides. Mutation analysis showed that all 4 available affected members harbored the A1555G mutation in MT-RNR1.

\section{Materials and methods Patients}

We identified a large Thai family with 11 members reported to have postlingual deafness after receiving aminoglycosides. We obtained and examined blood samples from 5 family members; 4 of whom were deaf (Figure 1). On examination, III-1, III-3, III-4, III-5 and IV-1 were 59, 56, 55, 53, and 12 years of age, respectively. The first 4 members had severe sensorineural hearing loss immediately after they received unknown medications to treat infections at the age of $9,3,8$, and 8 years, respectively. We obtained detailed informed consent statements signed by each participant. Written informed consent was obtained from the parents of the 12-year-old boy. This case report was specifically exempted from review by the Institutional Review Board of the Faculty of Medicine, Chulalongkorn University.

\section{MT-RNR1 gene analysis}

Six milliliters of peripheral blood was obtained from each individual after written informed consent was obtained. Genomic DNA was isolated from white blood cells using a QIAamp DNA blood mini kit according to the manufacturer's instruction (Qiagen, Valencia, CA, USA). Polymerase chain reaction (PCR)-amplification of the MT-RNR1 from genomic DNA was performed using primers MTRNR1-F 5'-
TGG CCA CAG CAC TTA AAC AC- ${ }^{\prime}$ and MTR NR1-R 5'-ACT ATA TCT ATT GCG CCA GG-3. We used 50 ng of genomic DNA, 1× PCR buffer (Fermentas, Thermo Fisher Scientific, Waltham, MA, USA), $1.5 \mathrm{mM} \mathrm{MgCl}, 0.2 \mathrm{mM}$ dNTPs, $0.2 \mu \mathrm{M}$ of each primer, and $0.5 \mathrm{U}$ of Taq polymerase (Fermentas) in a total volume of $20 \mu$ using the following parameters: $94^{\circ} \mathrm{C}$ for $3 \mathrm{~min}, 35$ cycles of $94^{\circ} \mathrm{C}$ for $45 \mathrm{~s}, 62^{\circ} \mathrm{C}$ for $45 \mathrm{~s}$, and $72^{\circ} \mathrm{C}$ for $90 \mathrm{~min}$, followed by $72^{\circ} \mathrm{C}$ for $5 \mathrm{~min}$. The PCR product of III-5 was treated with ExoSAP-IT (USP Corporation, Cleveland, OH, USA), according to the company recommendations, and sent for Sanger sequencing (Macrogen Inc, Seoul, Korea). Sequence data were analyzed using a Sequencer (version 4.2; Gene Codes Corporation, Ann Arbor, MI, USA).

The presence of the A1555G mutation in other family members was determined by digestion of the PCR products with the restriction enzyme BsmAI (New England Biolabs, Beverly, MA), according to the manufacturer's instructions. Digested DNA was analyzed by electrophoresis on $1 \%$ agarose gel using a Tris-borate buffer and visualized by Geldoc (Bio-Rad Laboratories Inc, Hercules, CA, USA).

\section{Results}

PCR-Sanger sequencing of III-5 revealed that the patient had a homoplasmic $A>G$ mutation at nucleotide position 1555 of the MT-RNR1 (Figure 2). PCR-restriction fragment length polymorphism (RFLP) showed that the other three affected members, III-1, III-3, and III-4, also harbored the homoplasmic A1555G mutation (Figure 3), while the unaffected IV-1 had only the wild-type nucleotide.

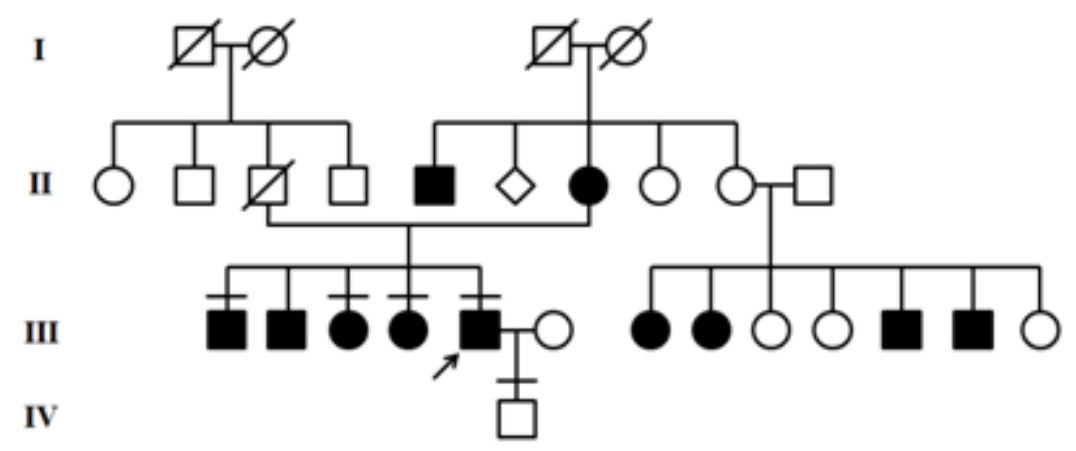

Figure 1. The pedigree of a Thai family with 11 members with aminoglycoside-induced deafness. An arrow, proband; blackened symbols, affected individuals; horizontal bars above symbols, individuals clinically examined. 
(a)

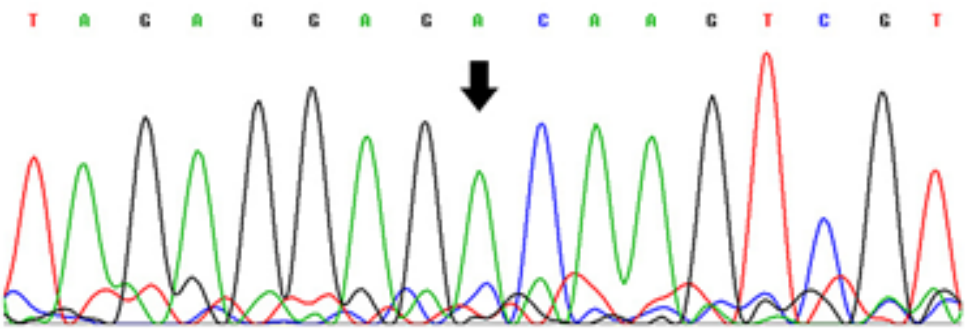

(b)

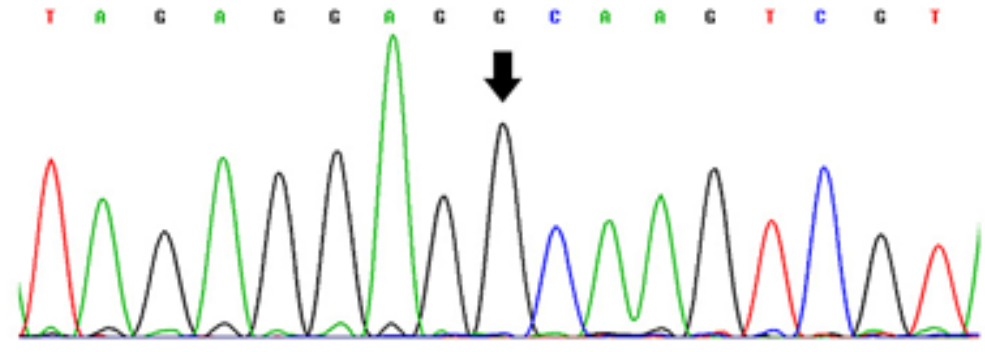

Figure 2. Mutation analysis of MT-RNR1. Electropherogram of MT-RNR1 from (A) a Thai healthy control and (B) III-5, which shows a homoplasmic $A>G$ mutation at position 1555 of the human mitochondrial DNA.

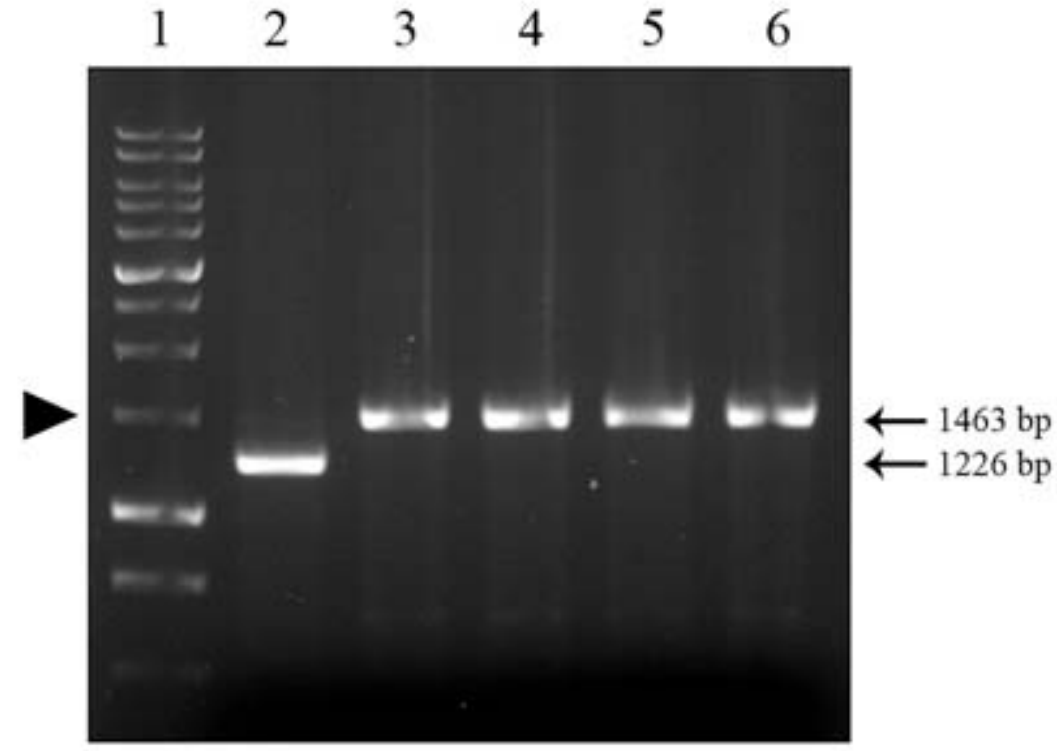

Figure 3. Restriction fragment length polymorphism (RFLP) analysis of MT-RNR1.

Lane 1 represents a 1000-bp marker with the 1500-bp band indicated by an arrow head. Lane 2 is a Thai healthy control showing a 1266-bp fragment, resulting from a restriction enzyme BsmAI digestion. Lanes 3-6 are III-1, III-3, III-4, and III-5 showing undigested 1463-bp PCR products, indicating that all 4 patients have a homoplasmic A1555G mutation.

\section{Discussion}

In our study, we first analyzed the A1555G mutation in the MT-RNR1 gene by PCR-Sanger sequencing in our proband. Then we performed RFLP analysis to determine the presence of this mutation in other family members. The mutation analyses showed that this family had the mitochondrial genetic susceptibility for aminoglycoside-induced deafness.

With a prevalence of almost 1 in 400 individuals in the British population [9], the A1555G mitochondrial variant could be considered common in some ethnic groups. As long as people with this genetic 
susceptibility are not given aminoglycosides, they could remain healthy with normal hearing throughout their entire life [9]. Nonetheless, there was an observation that the A1555G mutation could cause hearing loss even without exposure to aminoglycosides. The hearing loss in these cases tends to be later onset and less severe compared with those exposed to aminoglycosides [10]. By contrast, there has been a recent report of a child with a A1555G mutation who has normal hearing despite repeated exposure to aminoglycosides [11]. Factors affecting penetrance of this genetic susceptibility require further investigations.

Aminoglycosides produce their antibacterial effects by binding to the $30 \mathrm{~S}$ ribosomal subunit of bacterial ribosomes, which alters their conformation. This leads to codon misreading of RNA, induces errors in protein synthesis, and results in bacterial death [12]. Because structural differences lower the drug's affinity for eukaryotic ribosomes, aminoglycosides do not normally bind to human ribosomes, and therefore are generally safe for human use [4]. However, the mutation at position 1555 from adenine to guanine in human MT-RNR1 causes a structural rearrangement, which increases structural similarity of human mitochondrial rRNA to bacterial rRNA, promoting aminoglycoside binding. This can lead to a misreading in mitochondrial protein synthesis, which decreases mitochondrial ATP synthesis, compromises ion pump activity, and progressively decreases endocochlear potential, ultimately resulting in hearing loss [13].

Our present finding demonstrated that the A1555G mitochondrial mutation causing aminoglycoside-induced deafness, reported in several families worldwide, is also present in the Thai population. This genetic susceptibility is located within human mitochondrial DNA; therefore it is maternally inherited transferring from mothers to their children. Therefore, it is important that medical practitioners in Thailand be aware of this mitochondrial DNA mutation. This mutation should be considered before prescribing aminoglycosides, especially when there is deafness reported in a patient's family history. Patients who have this mutation should be prescribed with an alternative antibiotic to avoid this preventable deafness.

\section{Acknowledgments}

This study was supported by Royal Golden Jubilee Ph.D. Program (Grant No. PHD/0071/2552),
Ratchadapiseksomphot Endowment Fund of Chulalongkorn University (RES560530177-HR), and the Thailand Research Fund.

None of the authors have any conflict of interests to declare.

\section{References}

1. Mehl AL, Thomson V. The Colorado newborn hearing screening project, 1992-1999: on the threshold of effective population-based universal newborn hearing screening. Pediatrics. 2002; 109:E7.

2. Morton CC. Genetics, genomics and gene discovery in the auditory system. Hum Mol Genet. 2002; 11: 1229-40.

3. Schacht J, Talaska AE, Rybak LP. Cisplatin and aminoglycoside antibiotics: hearing loss and its prevention. Anat Rec (Hoboken). 2012; 295:1837-50.

4. Xie J, Talaska AE, Schacht J. New developments in aminoglycoside therapy and ototoxicity. Hear Res. 2011; 281:28-37.

5. del Castillo FJ, Rodriguez-Ballesteros M, Martin Y, Arellano B, Gallo-Teran J, Morales-Angulo C, et al. Heteroplasmy for the $1555 \mathrm{~A}>\mathrm{G}$ mutation in the mitochondrial 12S rRNA gene in six Spanish families with non-syndromic hearing loss. J Med Genet. 2003; 40:632-6.

6. Li R, Xing G, Yan M, Cao X, Liu XZ, Bu X, et al. Cosegregation of C-insertion at position 961 with the A1555G mutation of the mitochondrial 12S rRNA gene in a large Chinese family with maternally inherited hearing loss. Am J Med Genet A. 2004; 124A:113-7.

7. Yuan H, Qian Y, Xu Y, Cao J, Bai L, Shen W, et al. Cosegregation of the G7444A mutation in the mitochondrial COI/tRNA(Ser(UCN)) genes with the 12S rRNA A1555G mutation in a Chinese family with aminoglycoside-induced and nonsyndromic hearing loss. Am J Med Genet A. 2005; 138A:133-40.

8. Wang X, Lu J, Zhu Y, Yang A, Yang L, Li R, et al. Mitochondrial tRNAThr G15927A mutation may modulate the phenotypic manifestation of ototoxic 12S rRNA A1555G mutation in four Chinese families. Pharmacogenet Genomics. 2008; 18:1059-70.

9. Rahman S, Ecob R, Costello H, Sweeney MG, Duncan AJ, Pearce K, et al. Hearing in 44-45 year olds with $\mathrm{m} .1555 \mathrm{~A}>\mathrm{G}$, a genetic mutation predisposing to aminoglycoside-induced deafness: a population based cohort study. BMJ Open. 2012; 2:e000411.

10. Estivill X, Govea N, Barcelo E, Badenas C, Romero E, 
Moral L, et al. Familial progressive sensorineural deafness is mainly due to the mtDNA A1555G mutation and is enhanced by treatment of aminoglycosides. Am J Hum Genet. 1998; 62:27-35.

11. Al-Malky G, Suri R, Sirimanna T, Dawson SJ. Normal hearing in a child with the m.1555A>G mutation despite repeated exposure to aminoglycosides. Has the penetrance of this pharmacogenetic interaction been overestimated? Int J Pediatr Otorhinolaryngol. 2014; 78:969-73.
12. Kaul M, Barbieri CM, Pilch DS. Aminoglycosideinduced reduction in nucleotide mobility at the ribosomal RNAA-site as a potentially key determinant of antibacterial activity. J Am Chem Soc. 2006; 128: 1261-71.

13. Huth ME, Ricci AJ, Cheng AG. Mechanisms of aminoglycoside ototoxicity and targets of hair cell protection. Int J Otolaryngol. 2011; 2011:937861. 